\title{
El Gasto Sanitario Público en Castilla-La Mancha, 1995-2008: comparación con el conjunto de las Comunidades Autónomas.
}

\author{
Juan Simó Miñanaa
}

\begin{abstract}
a Médico de Familia. Centro de salud de Ansoain (Navarra). Plataforma 10 minutos.

Correspondencia: Juan Simó Miñana, Avda. Pío XII, $\mathrm{n}^{\circ} 6,8^{\circ}$ B, 31008 - Pamplona (Navarra, España). Telf.: 655678603, e-mail: ju.simom@gmail.com.
\end{abstract}

Recibido el 22 de diciembre de 2010.

Aceptado para su publicación el 26 de marzo de 2011.

\section{RESUMEN}

Objetivo. Conocer el crecimiento y reparto del gasto público (GSP) entre 1995 y 2008 según sectores en la sanidad pública de Castilla-La Mancha y su comparación con el conjunto de las 17 comunidades autónomas (CCAA).

Diseño. Estudio longitudinal retrospectivo.

Participantes. Las 17 CCAA.

Mediciones. Se determina el reparto del GSP castellano-manchego entre sus principales sectores y su crecimiento anual, comparándolo con la media autonómica. Se consideran también variables sociodemográficas como la renta y el envejecimiento poblacional.

Resultados. Entre 1995 y 2008, el GSP per cápita castellano-manchego es un 7\% inferior a la media autonómica, pero crece un $8 \%$ más. El gasto farmacéutico per cápita castellanomanchego es un $7 \%$ mayor que la media y su peso en el GSP es un $15 \%$ mayor. En CastillaLa Mancha, el incremento anual del gasto en atención hospitalaria es un 38\% mayor que el de atención primaria $(4,292 \%$ vs. $3,109 \%)$ y el hospital aumenta su participación en el GSP castellano-manchego un 0,079\% anual, pero la atención primaria la reduce un 0,695\%, un comportamiento similar al del conjunto autonómico. En términos per cápita, y en el promedio del periodo, el gasto hospitalario castellano-manchego es un $17 \%$ inferior a la media y el gasto en atención primaria un $6 \%$ superior.

Conclusiones. Entre 1995 y 2008, y a pesar de exhibir un GSP per cápita inferior al promedio autonómico, Castilla-La Mancha mantiene su atención primaria con ventaja presupuestaria respecto al promedio autonómico y respecto al propio sector hospitalario castellano-manchego. Palabras Claves. Servicios de salud, Gastos en Salud, Atención Primaria de Salud.

\section{ABSTRACT}

Public Health Expenditure in Castilla-La Mancha, 1995-2008: compared with that of all the Autonomous Communities

Objective. To determine the growth and the distribution of public health expenditure (PHE) among health sectors in Castilla-La Mancha (CLM) from 1995 to 2008 and to compare them with those of all the 17 Autonomous Communities (ACs).

Design. A longitudinal and retrospective study.

Participants. The 17 ACs.

Measurements. The distribution of the PHE among CLM health sectors and its annual growth are determined and compared to the AC average.. Demographic variables were also considered such as income level and population ageing.

Results. Between 2002 and 2008, the CLM PHE per capita was 7\% lower than the AC average but increased by $8 \%$. The CLM pharmaceutical expenditure per capita was $7 \%$ higher than the AC average and its share in the PHE was $15 \%$ higher. The annual growth of hospital care expenditure in CLM was $38 \%$ higher than that of primary care $(4.292 \%$ versus $3.109 \%)$ and the share of hospital care in the CLM PHE grew by $0.079 \%$ annually but the primary care decreased by $0.695 \%$, similar to that of the ACs. On average, over this period, the hospital care expenditure per capita in CLM was $17 \%$ lower than the AC average but the per capita expenditure in primary care was $6 \%$ higher.

Conclusions. Between 1995 and 2008, in spite of having a PHE per capita lower than the AC average, CLM maintained its primary care with a budgetary advantage compared to the AC average and to the CLM the hospital care expenditure.

Key words. Health Services, Health Expenditures, Primary Health Care. 


\section{INTRODUCCIÓN}

Nuestraatención primaria se sitúa desde los primeros años 90 en el epicentro de la infrapresupuestación relativa de la sanidad pública española respecto a Europa ${ }^{1}$ y el reparto de fondos públicos y su crecimiento entre los principales sectores de la sanidad pública entre 1995 y 2006 fue muy desfavorable para nuestra atención primaria ${ }^{2,3}$. Esta situación parece que empieza a corregirse ${ }^{4}$ tras la aprobación en 2006 por el Consejo Interterritorial del Proyecto AP- $21^{5}$, cuya estrategia $n^{\circ} 36$ recomienda incrementar los recursos financieros destinados a la atención primaria por encima del incremento de la media de los presupuestos sanitarios públicos. Por otro lado, cuando se comparan los sistemas sanitarios autonómicos, la sanidad pública de la Comunidad de Castilla-La Mancha resulta situada entre las mejores posiciones ${ }^{6}$, pero su atención primaria queda en una posición intermedia ${ }^{7}$.

El objetivo del presente trabajo es conocer el reparto de los fondos públicos para la sanidad pública castellano-manchega entre sus diferentes sectores (atención hospitalaria-especializada, primaria y fármacos), su crecimiento y su comparación con el conjunto de las 17 comunidades autónomas (CC. AA.) durante el periodo 1995 - 2008.

\section{MATERIAL Y MÉTODO}

El gasto sanitario público que se analiza en este trabajo incluye únicamente el de las CC.AA. Esto es, el gasto sanitario ocasionado por la asistencia sanitaria transferida a las CC.AA. y que representa actualmente más del $90 \%$ del gasto sanitario público. Por lo tanto, no se incluye el gasto sanitario público de la Administración Central del Estado, el de las mutualidades de funcionarios, el de las mutualidades de accidentes de trabajo, el de las corporaciones locales ni el de las ciudades autónomas de Ceuta y Melilla. Los datos de gasto sanitario público proceden de las Cuentas Satélite del Gasto Sanitario Público ${ }^{8,9}$. Proceden del INE los datos de población, producto interior bruto (PIB) y deflactor del $\mathrm{PIB}^{10-13}$.

Las variables expresan el promedio entre 1995 y 2008 para cada CC.AA. y son las siguientes: población (número de habitantes), PIB per cápita
(PIBpc), porcentaje de población $\geq 65$ años (P65), gasto sanitario público per cápita (GSpc), gasto hospitalario-especializada per cápita (GHpc), gasto en atención primaria per cápita (GAPpc), gasto farmacéutico (recetas del SNS) per cápita (GFpc), gasto en personal per cápita (GPpc), gasto en personal hospitalario-atención especializada per cápita (GPHpc), gasto en personal de atención primaria per cápita (GPAPpc), gasto hospitalarioatención especializada como porcentaje del gasto sanitario ( $\mathrm{GH} \% \mathrm{GS})$, gasto en atención primaria como porcentaje del gasto sanitario (GAP\%GS), gasto farmacéutico como porcentaje del gasto sanitario (GF\%GS) y gasto de personal como porcentaje del gasto sanitario (GP\%GS).

Los gastos per cápita y la renta per cápita se expresan en euros corrientes. Para obtener el crecimiento real de los gastos se deflacta su crecimiento nominal mediante el deflactor del PIB del periodo.

\section{RESULTADOS}

La tabla 1 muestra la distribución de las variables del estudio entre las CA.AA. La tabla 2 muestra el incremento anual real de la renta (PIB per cápita) y de algunos gastos durante el periodo de estudio. La tabla 3 muestra la variación anual del envejecimiento, de la población y de la participación de algunos sectores funcionales en el gasto sanitario público en el periodo de estudio. En relación con el promedio de las CCAA, se muestra el perfil evolutivo entre 1995 y 2008 de la renta y gastos sanitarios per cápita castellano-manchegos (figura 1), del envejecimiento poblacional castellano-manchego y del peso de los principales sectores funcionales dentro del gasto sanitario público de Castilla-La Mancha (figura 2).

La población castellano-manchega pasó de 1.697.263 habitantes en 1995 a 2.043.100 en 2008, lo que significa un incremento acumulado del $20,4 \%$ (un $1,441 \%$ anual) (tabla 3 ). Durante el mismo periodo, la población española aumentó un $17,2 \%$ (un 1,230\% anual). La proporción de habitantes de 65 o más años durante el periodo 1995-2008 pasó del $18,15 \%$ al $17,72 \%$, lo que significa una reducción acumulada del $2,4 \%$ del envejecimiento poblacional (un $0,162 \%$ anual) (tabla 3 ), mientras 


\begin{tabular}{|c|c|c|c|c|c|c|c|c|c|c|c|c|c|c|}
\hline & Población & P65 & $\mathrm{PIBpc}{ }^{\mathrm{a}}$ & $\mathrm{GSpc}^{\mathrm{a}}$ & $\mathrm{GHpc}^{\mathrm{a}}$ & GAPpc ${ }^{a}$ & GFpc $^{a}$ & $\mathrm{GPpc}^{\mathrm{a}}$ & $\mathrm{GPHpc}^{\mathrm{a}}$ & GPAPpc ${ }^{a}$ & GH\%GS & GAP $\%$ GS & GF\%GS & GP\%GS \\
\hline Andalucía & 7.552 .051 & 14,3 & 12.993 & 808 & 442 & 131 & 186 & 392 & 267 & 103 & 55,0 & 15,8 & 23,3 & 49,0 \\
\hline Aragón & 1.228 .291 & 20,7 & 18.491 & 926 & 504 & 134 & 204 & 474 & 320 & 114 & 54,2 & 14,7 & 22,2 & 51,6 \\
\hline Asturias & 1.078 .895 & 21,2 & 14.963 & 933 & 534 & 119 & 210 & 452 & 320 & 100 & 57,4 & 12,8 & 22,5 & 48,8 \\
\hline Baleares & 895.465 & 14,3 & 19.875 & 781 & 436 & 105 & 148 & 387 & 270 & 90 & 55,2 & 13,7 & 19,6 & 49,6 \\
\hline Canarias & 1.807 .451 & 11,7 & 15.871 & 888 & 481 & 110 & 175 & 389 & 266 & 95 & 53,3 & 12,4 & 19,7 & 43,5 \\
\hline Cantabria & 545.724 & 18,6 & 16.620 & 972 & 542 & 115 & 185 & 474 & 318 & 94 & 56,7 & 11,6 & 19,2 & 49,1 \\
\hline Castilla y León & 2.502 .093 & 22,0 & 16.107 & 878 & 444 & 148 & 190 & 448 & 275 & 130 & 50,6 & 17,3 & 21,6 & 51,7 \\
\hline Castilla-La Mancha & 1.810 .702 & 19,1 & 13.584 & 828 & 399 & 134 & 203 & 396 & 249 & 113 & 48,0 & 16,5 & 24,9 & 48,1 \\
\hline Cataluña & 6.573 .429 & 16,7 & 20.651 & 865 & 466 & 137 & 194 & 243 & 119 & 103 & 53,6 & 15,9 & 22,6 & 27,9 \\
\hline Comunidad Valenciana & 4.364 .631 & 16,1 & 15.973 & 811 & 413 & 108 & 220 & 342 & 224 & 91 & 50,7 & 13,5 & 27,3 & 43,2 \\
\hline Extremadura & 1.076 .406 & 18,6 & 11.319 & 909 & 449 & 157 & 204 & 440 & 274 & 134 & 49,6 & 17,5 & 22,7 & 48,9 \\
\hline Galicia & 2.745 .526 & 20,4 & 13.854 & 878 & 477 & 105 & 217 & 383 & 264 & 93 & 54,4 & 12,1 & 24,2 & 43,9 \\
\hline Madrid & 5.522 .685 & 14,3 & 22.877 & 801 & 499 & 98 & 143 & 401 & 289 & 81 & 62,3 & 12,3 & 17,8 & 50,4 \\
\hline Murcia & 1.228 .456 & 13,9 & 14.444 & 861 & 471 & 108 & 198 & 385 & 268 & 91 & 54,2 & 12,9 & 23,2 & 45,4 \\
\hline Navarra & 564.413 & 17,6 & 21.830 & 1.003 & 571 & 159 & 183 & 524 & 365 & 123 & 56,9 & 16,0 & 18,0 & 52,7 \\
\hline País Vasco & 2.112 .201 & 17,3 & 21.502 & 977 & 557 & 147 & 186 & 496 & 346 & 116 & 57,3 & 15,2 & 18,9 & 51,5 \\
\hline Rioja, La & 282.318 & 18,8 & 19.110 & 956 & 479 & 122 & 187 & 448 & 298 & 103 & 50,7 & 13,1 & 20,6 & 48,6 \\
\hline \multicolumn{2}{|c|}{ Promedio CCAA } & 17,4 & 17.063 & 887 & 480 & 126 & 190 & 416 & 278 & 104 & 54,1 & 14,3 & 21,7 & 47,3 \\
\hline \multicolumn{2}{|l|}{$\mathrm{DE}$} & 2,9 & 3448 & 68 & 49 & 19 & 21 & 66 & 55 & 15 & 3,5 & 1,9 & 2,6 & 5,8 \\
\hline \multicolumn{2}{|l|}{ CV } & 0,17 & 0,20 & 0,08 & 0,10 & 0,15 & 0,11 & 0,16 & 0,20 & 0,15 & 0,07 & 0,14 & 0,12 & 0,12 \\
\hline
\end{tabular}

Tabla 1. Distribución de las principales variables del estudio entre las comunidades autónomas (CCAA). Valores promedio del periodo 1995-2008 ( $n=17$ ). Población: número de habitantes; P65: Porcentaje (\%) de población con 65 o más años; PIBpc: Producto Interior Bruto per cápita (pc); GSpc: gasto sanitario pc; GHpc: gasto hospitalario-especializada pc; GAPpc: gasto en atención primaria pc; GFpc: gasto farmacéutico (recetas SNS) pc; GPpc: gasto en personal pc; GPHpc: gasto en personal hospitalario-especializada pc; GPAPpc: gasto en personal de atención primaria pc; GH\%GS: gasto hospitalario-especializada como \% del gasto sanitario; GAP\%GS: gasto en atención primaria como \% del gasto sanitario; GF\%GS: gasto farmacéutico como \% del gasto sanitario; GP\%GS: gasto en personal como $\%$ del gasto sanitario. DE: desviación estándar. CV: coeficiente de variación. a Unidades monetarias: euros corrientes.

\begin{tabular}{|c|c|c|}
\hline & \multicolumn{2}{|c|}{$\begin{array}{l}\text { Incremento anual real (\%) } \\
\text { Periodo 1995-2008 }\end{array}$} \\
\hline & Castilla-La Mancha & Promedio CC.AA. \\
\hline Renta per cápita (PIB per cápita) & 1,659 & 2,306 \\
\hline Gasto sanitario público per cápita & 4,106 & 3,812 \\
\hline Gasto hospitalario-especializada per cápita & 4,292 & 3,949 \\
\hline Gasto en Atención Primaria per cápita & 3,109 & 3,286 \\
\hline Gasto de personal per cápita & 3,620 & 3,243 \\
\hline Gasto de personal hospitalario-especializada per cápita & 3,824 & 3,502 \\
\hline Gasto de personal en atención primaria per cápita & 2,833 & 2,796 \\
\hline Gasto farmacéutico (recetas SNS) per cápita & 3,686 & 3,797 \\
\hline
\end{tabular}

Tabla 2. Incremento anual real ${ }^{\mathrm{a}}$ de la renta y algunos gastos sanitarios públicos per cápita. Castilla-La Mancha comparada con el promedio de las 17 Comunidades Autónomas (CC.AA.). Periodo 1995-2008. ${ }^{\text {a }}$ Incremento real anual = incremento nominal anual - incremento anual del deflactor del PIB. 


\begin{tabular}{|c|c|c|}
\hline & \multicolumn{2}{|c|}{$\begin{array}{l}\text { Variación anual (\%) } \\
\text { Periodo 1995-2008 }\end{array}$} \\
\hline & Castilla-La Mancha & Promedio CC.AA. \\
\hline Gasto hospitalario-especializada como $\%$ del gasto sanitario público & 0,079 & 0,152 \\
\hline Gasto en atención primaria como \% del gasto sanitario público & $-0,695$ & $-0,504$ \\
\hline Gasto farmacéutico como \% del gasto sanitario público & $-0,230$ & $-0,038$ \\
\hline Gasto de personal como \% del gasto sanitario público & $-0,156$ & $-0,531$ \\
\hline Envejecimiento ( $\%$ de población con 65 o más años) & $-0,162$ & 0,598 \\
\hline Población (número de habitantes) & 1,441 & 1,230 \\
\hline
\end{tabular}

Tabla 3. Variación anual (en porcentaje) de la población, del envejecimiento poblacional y de la participación de algunos sectores en el gasto sanitario público. Castilla-La Mancha comparada con el promedio de las 17 Comunidades Autónomas (CC.AA.). Periodo 1995-2008.

que en el conjunto de la población española durante el mismo periodo aumentó el envejecimiento (7,9\% acumulado, 0,598\% anual).

Durante el periodo 1995-2009, el crecimiento del gasto sanitario público en Castilla-La Mancha es un $8 \%$ superior al promedio autonómico $(4,106 \%$ vs. $3,812 \%$ ) (tabla 2). Se detectan diferencias relevantes en el crecimiento de los principales sectores funcionales del gasto sanitario público castellano- manchego. Concretamente, el incremento del gasto en atención hospitalaria es un $38 \%$ mayor que el de atención primaria $(4,292 \%$ vs. $3,109 \%)$ (tabla 2). También, el incremento del gasto en personal de hospital es un $35 \%$ mayor que el del gasto en personal de atención primaria $(3,824 \%$ vs. $2,833 \%)$ (tabla 2). El gasto en atención hospitalaria es el que más crece tanto en Castilla-La Mancha como en el conjunto de las autonomías (3,949\%) (tabla 2). El gasto en personal de atención primaria es el que

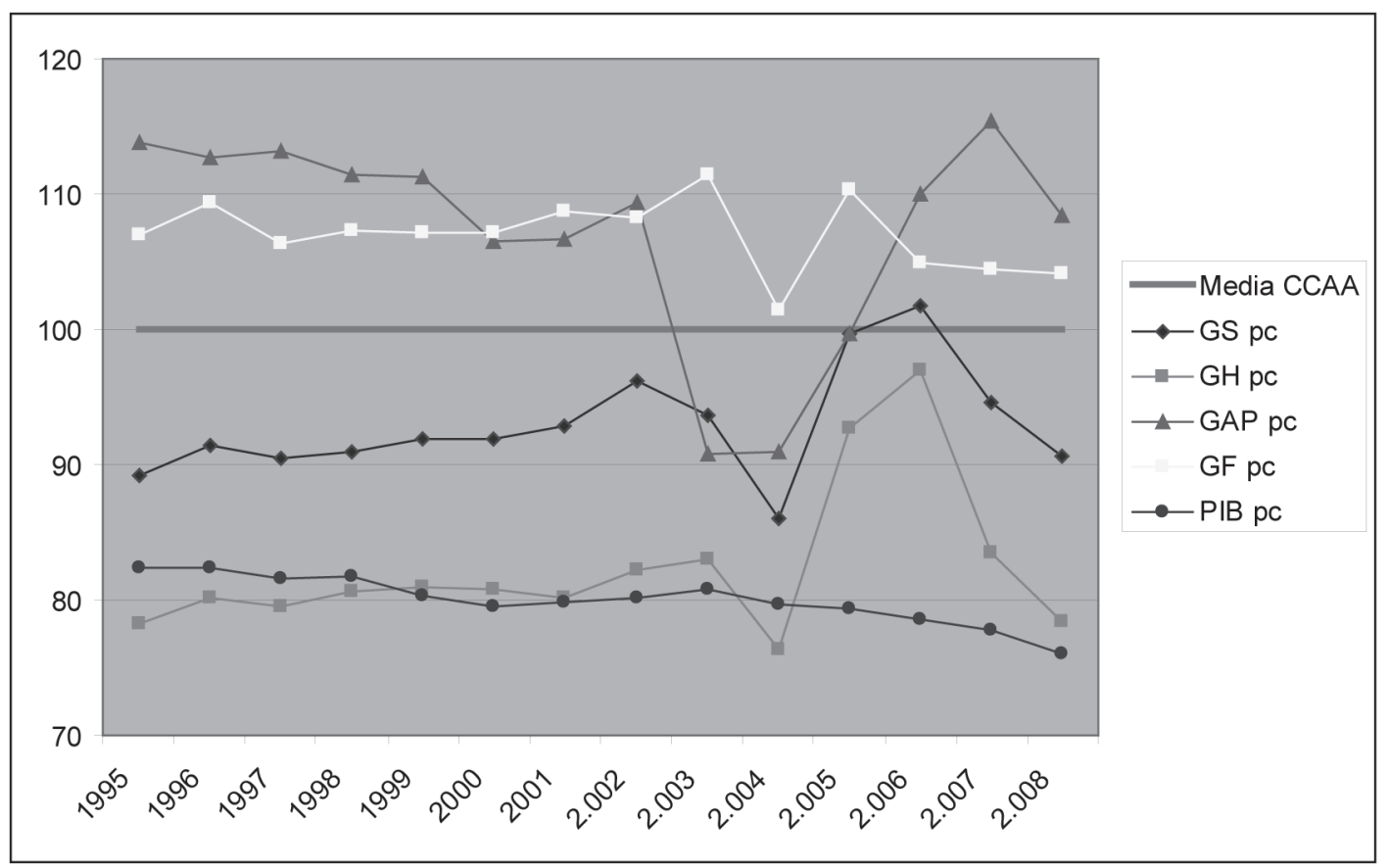

Figura 1.- Perfil evolutivo entre 1995 y 2008 de la renta per cápita y gastos sanitarios en términos per cápita en Castilla-La Mancha comparados con el promedio de las 17 comunidades autónomas. Media CCAA: Promedio comunidades autónomas; PIBpc: Producto Interior Bruto per cápita (pc); GSpc: gasto sanitario pc; GHpc: gasto hospitalario-especializada pc; GAPpc: gasto en atención primaria pc; GFpc: gasto farmacéutico (recetas SNS) pc. 


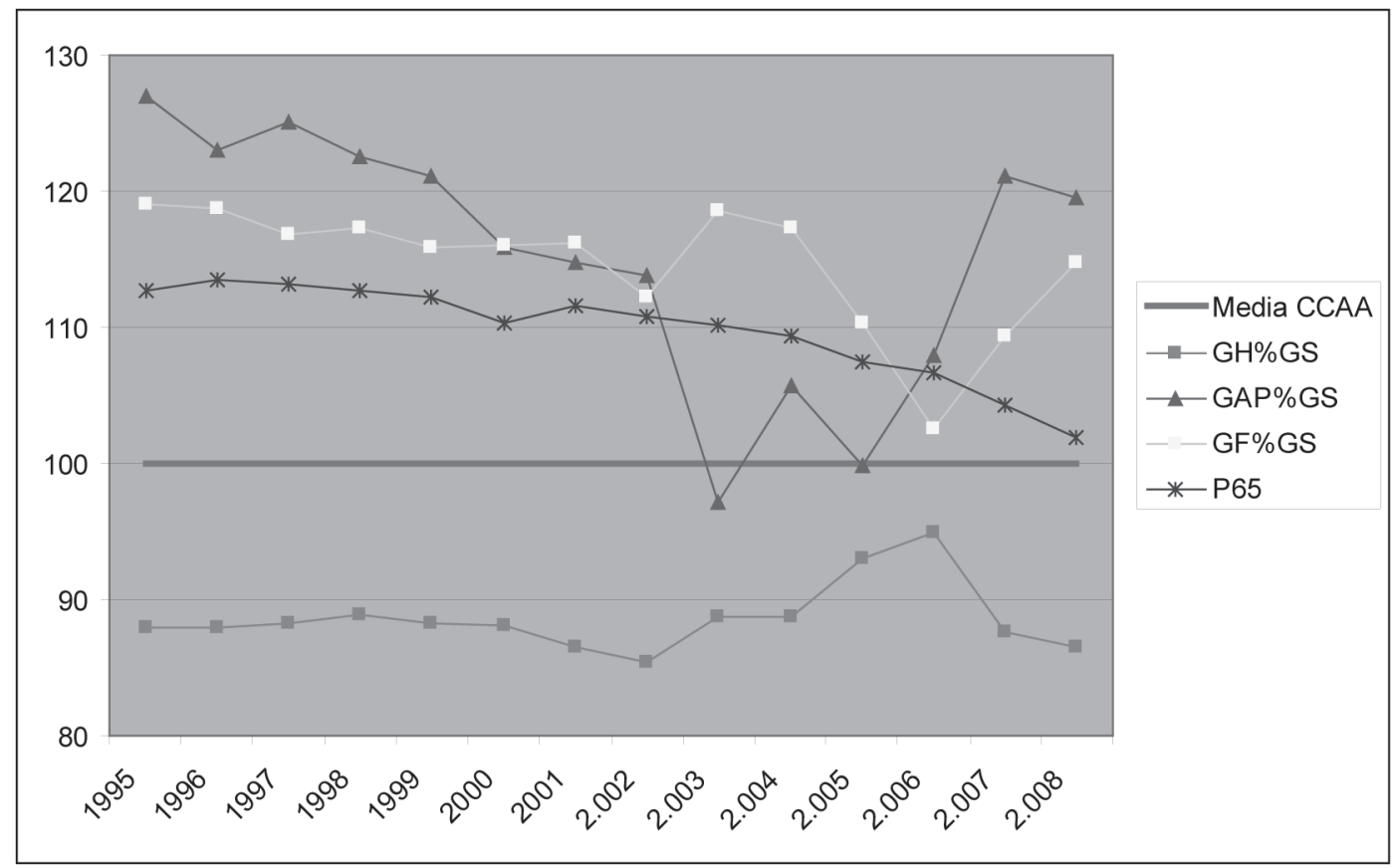

Figura 2.- Perfil evolutivo entre 1995 y 2008 del envejecimiento poblacional y de los gastos sanitarios expresados como parte del sanitario público en Castilla-La Mancha comparados con el promedio de las 17 comunidades autónomas. Media CCAA: Promedio comunidades autónomas; GH\%GS: gasto hospitalario-especializada como \% del gasto sanitario; GAP\%GS: gasto en atención primaria como \% del gasto sanitario; GF\%GS: gasto farmacéutico como \% del gasto sanitario; P65: Porcentaje (\%) de población con 65 o más años.

menos crece en Castilla-La Mancha y también en el conjunto autonómico $(2,796 \%)$ (tabla 2$)$. Todos los gastos crecen por encima de la renta tanto en Castilla-La Mancha como en el conjunto autonómico (tabla 2).

El hospital es el único sector que aumenta su participación en el gasto sanitario público de Castilla-La Mancha (un 0,079\% anual), mientras que la atención primaria y la farmacia de receta lo reducen anualmente un $0,695 \%$ y un $0,230 \%$, respectivamente. También reduce su participación en el gasto sanitario público castellano-manchego el gasto de personal (un 0,156\% anual) (tabla 3). Del mismo modo, en el conjunto autonómico todos los sectores reducen su participación excepto el sector hospitalario que la aumenta (tabla 3 ).

Las figuras 1 y 2 nos muestran el perfil evolutivo, el skyline presupuestario, desde 1995 hasta 2008, del gasto sanitario público castellano-manchego total y desagregado por sectores funcionales comparado con el promedio autonómico. Respecto al perfil de los gastos per cápita (figura 1), se comprueba que el gasto per cápita en atención primaria discurre durante casi todo el periodo por encima del promedio autonómico. En cambio, el gasto per cápita hospitalario es siempre inferior a dicho promedio, la mayor parte de los años un $20 \%$ inferior. El gasto farmacéutico per cápita es durante todo el periodo un $5-10 \%$ superior a la media autonómica. En cuanto al peso de los distintos sectores en el gasto sanitario público castellano-manchego (figura 2), prácticamente se repite el mismo perfil. El peso del sector hospitalario es inferior en aproximadamente un $10 \%$ al promedio autonómico durante todo el periodo y el peso de la atención primaria y el de la farmacia de receta son ambos superiores a dicho promedio.

\section{DISCUSIÓN}

Se ha propuesto la utilización de un deflactor específico del gasto sanitario público nominal en lugar del recurso habitual al deflactor del $\mathrm{PIB}^{14}$. Aunque la construcción de un deflactor especifico para el gasto sanitario público nominal no está exenta de dificultades metodológicas, disponemos en la literatura de una propuesta ${ }^{15}$ en la que sus autores estimaron un deflactor específico del gasto 
sanitario público español para el periodo 19861994. El deflactor específico del gasto sanitario público estimado por los autores creció durante este periodo a una tasa anual media del $4,91 \%$, mientras que el deflactor del PIB lo hizo a una tasa media del $6,02 \%$. Por tanto, la utilización del deflactor del PIB infraestimaría levemente el crecimiento real acumulado del gasto sanitario público durante el periodo 1986-1994. No disponemos de un deflactor específico del gasto sanitario público que podamos utilizar para el periodo de estudio del presente trabajo (1995-2008). No obstante, es posible, tal y como ocurría durante el periodo 1986-1994, que la utilización del deflactor del PIB infraestime levemente el crecimiento real del gasto sanitario público durante el periodo 1995-2008. Sin embargo, es improbable que esa eventual infraestimación invalide los principales resultados y conclusiones de este trabajo.

El gasto de personal en la sanidad pública española fue la diana del "control" del gasto durante el periodo $1995-2006^{2,3}$ y también cuando ampliamos el periodo de análisis hasta 2008. En el conjunto autonómico el gasto de personal es el segundo que menos crece tras el gasto en atención primaria, crece un $18 \%$ menos que el gasto sanitario (tabla 2) y es el que más reduce su participación en el gasto (tabla 3). En Castilla-La Mancha, también es el gasto de personal el segundo que menos crece tras el de atención primaria y crece un $16 \%$ menos que el gasto sanitario (tabla 2). Pero en contraste con lo que ocurre en el conjunto autonómico, el gasto farmacéutico en Castilla-La Mancha crece prácticamente lo mismo que el gasto en personal (tabla 2) y reduce su participación en el gasto incluso más que lo hace el gasto de personal (tabla 3 ).

En Castilla-La Mancha, el gasto en atención hospitalaria aumenta un $38 \%$ más que el de atención primaria y el gasto en personal de atención hospitalaria crece un $35 \%$ más que el gasto en personal de atención primaria (tabla 2). Esto tiene su reflejo en la participación de ambos sectores en el gasto sanitario público castellanomanchego: la atención hospitalaria aumenta ligeramente su participación, pero la atención primaria la reduce de forma importante (tabla 3). De hecho, la atención primaria es el sector que más reduce su participación en el gasto sanitario público castellano-manchego y la reduce más que el promedio autonómico (tabla 3). Pese a ello, y en relación con el promedio autonómico, la atención primaria en Castilla-La Mancha no resulta presupuestariamente discriminada frente al propio hospital castellano-manchego, pues la misma parte de una mejor posición que éste en relación con dicho promedio, ventaja presupuestaria que, además, mantiene durante todo el periodo analizado (figuras 1 y 2 ).

Al estudiar la relación de la renta y el envejecimiento poblacional con el presupuesto de los distintos sectores funcionales entre las CC.AA. ${ }^{2-4,16,17}$, se observa una tendencia a que las comunidades más ricas gasten menos per cápita en farmacia de receta y más en atención hospitalariaespecializada, pero no más en atención primaria. Las comunidades más ricas muestran también un mayor peso del sector hospitalario dentro del gasto sanitario público, pero un menor peso de la farmacia de receta. Entre las comunidades más envejecidas, con mayor proporción de población con 65 a más años, se detecta un mayor gasto per cápita en farmacia y en atención primaria, pero no más en hospital, de modo que las más envejecidas tienden a mostrar un mayor peso de la atención primaria y de la farmacia de receta dentro de su gasto sanitario público. La Comunidad de CastillaLa Mancha, un 20\% por debajo de la renta media autonómica (tabla 1 y figura 1) y con una población un $10 \%$ más envejecida (tabla 1 y figura 2), es un ejemplo de estas observaciones. En consonancia con ello, en relación con la media autonómica y en el promedio del periodo, Castilla-La Mancha muestra un gasto farmacéutico como parte de su gasto sanitario público un $15 \%$ mayor (tabla 1) y un gasto farmacéutico per cápita un $7 \%$ mayor (tabla 1). Afortunadamente para su atención primaria, la sanidad pública castellano-manchega ha apostado por la inversión en este nivel de atención y, pese a exhibir un gasto sanitario público per cápita un $7 \%$ inferior a la media autonómica (tabla 1), Castilla-La Mancha ha privilegiado presupuestariamente a su atención primaria frente al hospital. Así, el gasto per cápita castellano-manchego en atención primaria en el promedio del periodo es un $6 \%$ superior a la media autonómica y el peso de la atención primaria en el gasto sanitario público castellano-manchego es también un $15 \%$ mayor que dicha media (tabla 
1). Por el contrario, su gasto per cápita hospitalario es un $17 \%$ menor que la media autonómica y el peso del hospital dentro del gasto sanitario público castellanomanchego es también un $11 \%$ inferior a dicha media (tabla 1).

\section{BIBLIOGRAFÍA}

1. Simó J, Gérvas J, Seguí M, De Pablo R, Domínguez J. El gasto sanitario en España en comparación con el de la Europa desarrollada, 1985-2001. La atención primaria española, Cenicienta europea. Aten Primaria. 2004; 34:472-81.

2. Simó J. El gasto sanitario en España, 1995-2002. La atención primaria, Cenicienta del Sistema Nacional de Salud. Aten Primaria. 2007; 39:127-32.

3. Simó J. Financiación del hospital y de la atención primaria españoles. Periodo 1995-2006. En: Palomo L (Coord.). Expectativas y realidades en la atención primaria española". Madrid: Fundación $1^{\circ}$ de Mayo y Ediciones GPS; 2010.

4. Simó J. El gasto sanitario en España, 2002-2008: ¿Empieza el rescate presupuestario de la atención primaria? Aten Primaria. (en prensa)

5. Marco Estratégico para la mejora de la Atención Primaria en España: 2007-2012. Proyecto AP-21. Madrid: Ministerio de Sanidad y Consumo; 2007.

6. Los servicios sanitarios de las CCAA. Informe 2010 (VII Informe). Septiembre 2010. Federación de Asociaciones para la Defensa de la Sanidad Pública (FADSP). Disponible en: http://www.nodo50.org/fadsp/pdf/INFORME.ccaa2010.doc.

7. Martín-García M, Sánchez-Bayle M, Palomo L. El desarrollo de la atención primaria en relación con la orientación política de los gobiernos autonómicos. Aten Primaria. 2008; 40:27784

8. Ministerio de Sanidad y Consumo. Gasto Sanitario Territorializado por Comunidades Autónomas (19882005). Serie histórica. Disponible en: http://www. msc.es/estadEstudios/estadisticas/docs/EGSP2006/ Serie_1988_2005_Nuevo.xls.
9. Cuentas Satélite del Gasto Sanitario Público. Tablas estadísticas. Estadística del gasto sanitario público. Principios de Devengo. (2002-2008). Evolución y clasificaciones por subsectores de gasto. Comunidades Autónomas. Disponible en: http://www.msps.es/ estadEstudios/estadisticas/docs/EGSP2008/egsp_gasto_ real.xls.

10. Instituto Nacional de Estadística. Cifras de población. Series históricas de población. Disponible en: http:// www.ine.es/jaxi/menu.do?type=pcaxis $\&$ path $=/ \mathrm{t} 20 / \mathrm{e} 245 /$ p05\&file=inebase.

11. Instituto Nacional de Estadística. Padrón municipal: explotación estadística y Nomenclátor. Explotación estadística del Padrón. Datos nacionales, por CCAA y por provincias. Población por sexo, comunidades y provincias y edad. Disponible en: http://www.ine.es/jaxi/menu.do?type= pcaxis\&path $=\% 2 \mathrm{Ft} 20 \% 2 \mathrm{Fe} 245 \&$ file $=$ inebase $\& \mathrm{~L}=0$.

12. Instituto Nacional de Estadística. Cuentas económicas. Contabilidad Regional de España. Producto interior bruto a precios del mercado. Tablas por comunidades autónomas. Serie 1995-2009. Disponible en: http://www.ine.es/daco/ daco42/cre00/serieh/cre00 sh.htm.

13. Instituto Nacional de Estadística. Producto Interior Bruto (PIB). Base 2000. Crecimiento en volumen. Disponible en: http://www.ine.es/prensa/pib_tabla_cne.htm.

14. Murillo C, Baró E. Los deflactores sanitarios públicos: construcción y seguimiento de las variaciones en las prestaciones sanitarias medias. Madrid: Ministerio de Sanidad y Consumo; 1999.

15. Blanco A, De Bustos A. El gasto sanitario público en España: diez años de Sistema Nacional de Salud. Hacienda Pública Española. 1996; 3(138):3-27.

16. Simó J. Evolución del gasto en sanidad pública y sus sectores durante el periodo 1999-2005: comparación entre Comunidades Autónomas. Observatorio Sanitario en Atención Primaria. Plataforma 10 minutos. Disponible en: http://www.observatoriosanitario.org/.

17. Simó J, Gérvas J. Gasto farmacéutico en España y en Europa (1995-2002): el 'despilfarro' español, un mito sin fundamento. Rev Adm Sanit. 2007; 5:1-15. 